\title{
Na? Sind Sie geimpft?
}

\author{
Winfried V. Kern
}

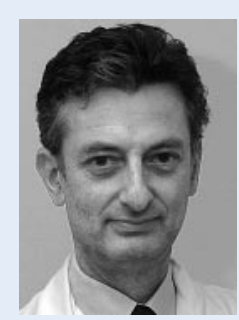

Winfried V. Kern

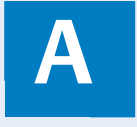

lle Jahre wieder müssen wir feststellen: Die Influenza-Impfrate beim medizinischen Personal ist erneut weit entfernt vom Ziel. Wir hatten vor einigen Jahren mal das Konzept „Influenza 50+“ dazu formuliert und mit Plakaten intensiv beworben. Abteilungen, in denen $>50 \%$ der Mitarbeiter gegen saisonale Influenza geimpft wurden, sollten ein Zertifikat sowie eine lobende Erwähnung in den internen News bekommen. Das Ergebnis war ernüchternd. Vier Abteilungen, darunter Klinische Infektiologie und Medizinische Mikrobiologie hatten es geschafft. Die Impfrate insgesamt war gegenüber den Vorjahren kaum angestiegen.

Dieses Jahr haben wir diskutiert, ob man einen Schritt weiter gehen soll und kann. Schriftliche Ablehnung des Impfangebotes („opt out“) wäre eine Möglichkeit aber, keine Chance! Man besteht darauf, die Evidenz und die Empfehlungen selbst zu interpretieren und in persönlicher Entscheidungsfreiheit sich und andere zu infizieren. Eine schöne Studie aus Atlanta hat gezeigt, dass dort „opt out“ die Impfrate von 43 auf $67 \%$ anhob [1]. In St. Louis und Seattle wurden im Rahmen eines Programms „Influenza-Pflichtimpfung“ Impfraten $>90 \%$ erzielt [2, 3]. Bei solchen Zahlen wird man richtig neidisch und muss an der eigenen Methodik zweifeln. Bei medizinischem Personal in Deutschland schätzt man seit vielen Jahren Influenza-Impfraten, die in der Größenordnung von 15-30\% stagnieren. Die Schweinegrippe hat die Impfmüdigkeit eher noch gefördert.

Was kann man tun? Hier gibt es einige Erfahrungen. Wichtig sind die arbeitsplatznahe Impfung und ein zeitlich flexibles Angebot hierfür. Wichtig ist auch die Information, aber nicht in Form von Postern/Flyern, sondern in Form von Vorträgen und Diskussionen, bei denen man überzeugt - nicht so sehr durch wissenschaftliche Daten, sondern durch überzeugende Rede, durch Erfahrung, durch Engagement in der Sache, durch vorbildliches Verhalten, durch Erzeugen eines sanften Drucks bei Ihren Zuhörern im Sinne von ,ich glaube, da müssen wir doch mal mitmachen“.

Probieren Sie es. Lassen Sie sich, Ihre Anverwandten, Ihre Mitarbeiter impfen. Impfen Sie Ihren Chefarzt und Verwaltungsleiter und seine Sekretärinnen und lassen Sie diese Aktionen fotografieren. Zeigen Sie die Fotos den „informationswilligen“ (noch) nicht geimpften Mitarbeitern. Zeigen Sie beim Gespräch durch Gesichtsausdruck, Körperhaltung und dieses „Etwas“ im Blick, dass Sie sich in der „Community der Geimpften“ zusammen mit dem Chef (!) sehr, sehr wohl fühlen und den Eindruck haben, dass die nächste InfluenzaSaison schon bald kommen kann ohne dem Betrieb und ihnen etwas anhaben zu können. Sie rechnen - ganz beiläufig - in der Tat damit, dass Sie die krankheitsfreien Tage zum noch fitter werden beim Skifahren verbringen können.

$\mathrm{Na}$ ? Sind Sie schon geimpft?

\section{Literatur}

1 Ribner BS, Hall C, Steinberg JP et al. Use of a mandatory declination form in a program for influenza vaccination of healthcare workers. Infect Control Hosp Epidemiol 2008; 29: $302-308$

2 Rakita RM, Hagar BA, Crome P et al. Mandatory influenza vaccination of healthcare workers: a 5-year study. Infect Control Hosp Epidemiol 2010; 31: 881 $-888$

3 Babcock HM, Gemeinhart N, Jones M et al. Mandatory influenza vaccination of health care workers: translating policy to practice. Clin Infect Dis 2010; 50: $459-464$ 